\title{
КИНЕТИКА И МЕХАНИЗМ ПРЕВРАЩЕНИЯ В ВОДОРОДНУЮ ПЛАЗМУ ПОВЕРХНОСТНО-АКТИВНОЙ ЖИДКОСТИ В ЗОНЕ РЕЗАНИЯ
}

Рассматриваются вопросы снижения энергозатрат на проиесс механической обработки металлов. Обсуждаются химические превращения СОТС, на конечном этапе которых создается углерод и водород в активных формах. Установлено, что в образиах, полученных в результате резания металла с применением данной технологии, регистрировался проиесс десорбиии водорода. Экспериментальный материал дает основание считать, что адсорбиия поверхностно-активной среды на металле, который деформируется, не является определяющим фактором в облегчении прочесса деформации, а только первым этапом преобразования среды.

Теплота является одним из основных физических показателей прочесса резания. Её основными источниками являются пластическая деформация, происходящая прежде всего в зоне максимальных сдвигов, и трение в зонах контакта инструмента со стружкой и заготовкой.

Превращение среды из физически активной в радикально активную обусловливается наложением $u$ взаимодействием нескольких «внутренних» $u$ «внешних» физических явлений, возникающих в зоне динамического контакта инструмента с обрабатываемым металлом.

Решаюшую роль в снижении прочности металлов решают активные формы водорода. Установлено, что вспомогательными факторами облегчения процесса резания является температура, эмиссия электронов, каталитически-активная поверхность разрушаемого металла действует 6 комплексе на поверхностно-активную жидкость, переводя ее в новое радикальное состояние водородную плазму.

Отмечено, что адсорбция поверхностно-активной среды на обрабатываемом металле, деформируется, не является конечным определяющим фактором в облегчении процесса деформации и разрушения при резании, а только первым, но очень важным элементом в системе многоступенчатых химических превращеенй технологической среды. При термодеструкции полимерной присадки СОТС на нагретых поверхностях металла, на промежуточных стадиях химических реакций образуются продукты, обладающие чрезвычайно высокой химической активностью, а именно: водород в активных формах, радикалы и относительно стабильные соединения.

Ключевые слова: Водород, десорбция, обработка металла, прочность, разрушение, поверхностно-активная среда, СОТС.

В.О. СОШКО

Херсонський національний технічний університет ORCID: 0000-0002-1788-0855

І.П. СІМІНЧЕНКО

Херсонський національний технічний університет ORCID: 0000-0001-7567-6062

\section{КІНЕТИКА І МЕХАНІЗМ ПЕРЕТВОРЕННЯ В ВОДНЕВІЙ ПЛАЗМІ ПОВЕРХНЕВО-АКТИВНОЇ РІДИНИ В ЗОНІ РІЗАННЯ}

Розглядаються питання зниження енерговитрат на прочес механічної обробки металів. Обговорюються хімічні перетворення МОТЗ, на кінцевому етапі яких створюється вуглець $i$ водень 8 активних формах. Встановлено, шьо в зразках, отриманих в результаті різання металу із застосуванням даної технології, реєструвався процес десорбиії водню. Експериментальний матеріал дає підставу вважати, щуо адсорбиія поверхнево-активного середовища на металі, деформується, не є визначальним фактором в полегшенні процесу деформації, а тільки першим етапом перетворення середовища.

Теплота є одним з основних фізичних показників процесу різання. Ї̈ основними джерелами $\epsilon$ пластична деформація, яка відбувається насамперед у зоні максимальних зрушень, $і$ тертя в зонах контакту інструменту зі стружкою $і$ заготовкою. 
Перетворення середовища з фізично активної в радикально активну обумовлюється накладенням і взаємодією декількох «внутрішніх» $i$ «зовнішніх» фізичних явищ, щзо виникають в зоні динамічного контакту інструменту з оброблюваним металом.

Вирішальну роль в зниженні міџності металів вирішують активні форми водню. Встановлено, щчо допоміжними факторами полегшення процесу різання $\epsilon$ температура, емісія електронів, каталітично активна поверхня руйнується металу діє в комплексі на поверхнево-активна рідина, переводячи ї̈ в нове радикальне стан - водневу плазму.

Відзначено, щчо адсорбиія поверхнево-активного середовища на металі, деформується, не $\epsilon$ кінцевим фактором в полегшенні процесу деформації і руйнування, а тільки першим, але дуже важливим елементом в системі багатоступеневих хімічних перетворень середовища. При термодеструкції полімерної присадки МОТЗ на нагрітих поверхнях металу, на проміжних стадіях хімічних реакиій утворюються продукти, щз володіють надзвичайно високою хімічною активністю, а саме: водень в активних формах, радикали і відносно стабільні з'єднання.

Ключові слова: Водень, десорбиія, обробка метала, міиність, руйнування, поверхнево-активне середовище, МОТЗ.

V.A. SOSHKO

Kherson National Technical University ORCID: 0000-0002-1788-0855

I.P. SIMINCHENKO

Kherson National Technical University ORCID: 0000-0001-7567-6062

\section{KINETICS AND MECHANISM OF TRANSFORMATION IN A HYDROGEN PLASMA OF A SURFACE-ACTIVE LIQUID IN THE CUTTING AREA}

The issues of reducing energy costs for the process of metal machining are considered. The chemical transformations of cuttinh emulsion are discussed, at the final stage of which carbon and hydrogen in active forms are created. It was found that in the samples obtained as a result of metal cutting using this technology, the process of hydrogen desorption was recorded. The experimental material gives reason to believe that the adsorption of a surface-active medium on a metal is deformed, is not a determining factor in facilitating the deformation process, but only the first stage of the transformation of the medium.

Heat is one of the main physical indicators of the cutting process. Its main sources are plastic deformation, which occurs primarily in the zone of maximum displacements, and friction in the zones of contact of the tool with the chips and the workpiece.

The transformation of the medium from physically active to radically active is due to the superposition and interaction of several "internal" and "external" physical phenomena that occur in the zone of dynamic contact of the tool with the metal being processed.

The decisive role in reducing the strength of metals is solved by the active forms of hydrogen. It has been established that auxiliary factors to facilitate the cutting process are temperature, electron emission, and the catalytically active surface of the metal being destroyed acts in complex on the surface-active liquid, transferring it to a new radical state - hydrogen plasma.

It is noted that the adsorption of a surface-active medium on a metal that is deformed is not the final factor in facilitating the process of deformation and fracture, but only the first, but very important element in the system of multi-stage chemical transformations of the medium. During thermal degradation of the polymer additive cuttinh emulsion on heated metal surfaces, at the intermediate stages of chemical reactions, products are formed that have extremely high chemical activity, namely hydrogen in active forms, radicals, and relatively stable compounds

Keywords: Hydrogen, desorption, metalworking, durability, fracture, surfactant, cutting emulsion.

\section{Постановка проблемы}

Экспериментальными исследованиями, результаты которых анализируются в работе [1], было установлено, что 1,0 - 1,5\% высокомолекулярного соединения (например, полиэтилена или поливинилхлорида) в смазочно-охлаждающее технологическое средство (СОТС), в его модельную жидкость, или в жидкость, которая является основой СОТС (вода, индустриальное масло), происходит многократное снижение энергозатрат на процесс механической обработки металлов резанием или давлением, а также изменение других параметров, характеризующих обрабатываемость металла.

Полученные данные имеют огромное практическое значение [2], так как отсюда следуют непосредственные рекомендации поиска эффективных присадок к СОТС среды полимерных соединений.

Такой необходимый шаг к созидательному изменению свойств твердого тела может быть сделан после раскрытия механизма скачкообразного изменения эффективности жидкости при добавлении в ее 
состав полимера, не изменяется ни ее химсостав, ни ее адсорбционная активность. Имеются также все основания полагать, что подобные исследования дадут возможность выявить скрытые резервы, заложенные в эффекте влияния среды на физические и механические свойства твердых тел, с целью наиболее полного их использования в технологических процессах обработки, с более высокой эффективностью и экономичностью.

\section{Анализ исследований и публикаций}

Прогресс в изучении твердых тел и представлений о влиянии на процессы их деформации и разрушения сред различной физической и химической активности, находящихся в различных фазовых состояниях, позволили выдвинуть гипотезу о пиролитических превращениях полимерной составляющей СОТС, которые могут осуществляться под влиянием температуры образующейся в процессе резания в зоне контакта режущего инструмента с обрабатываемым материалом [1]. При этом предполагалось что на последнем этапе химических преобразований СОТС, в составе которой содержаться высокомолекулярные цепи полимера, построенные, в основном, из атомов углерода и водорода (например, полиэтилен), будут образовываться активные формы углерода и водорода $\left(\mathrm{H}^{+}\right.$и $\left.\mathrm{H}^{-}\right)$. Это означает что исходная жидкость с несколькими десятками растворенных и эмульгированных в ней компонентов преобразуется в новое фазовое состояние - водородную плазму.

Формулирование цели исследования

Согласно предложенной гипотезы параллельно в химической активацией среды должна происходить перманентная карбонизация режущей кромки инструмента, а также наводороживание металла в области его пластической деформации перед кончиком микротрещины и активное участие водорода в термо-механо-химическом (ТМХО) процесса разрушения.

Все это позволяет считать, что решающая роль в изменении прочности металла под влиянием физически активной полимерсодержащей СОТС отводится на процессу физической адсорбции, как утверждается общепринятой теорией адсорбционного облегчения деформации и разрушения [3-6], а частицам водорода, обладающими электрическим зарядом.

В предположении, что эксперимент в будущем подтвердить справедливость выдвинутой гипотезы еe основные положения легли в основу разработки способа термо-механо-химической обработки (ТМХО) и создания различных рецептур СОТС, а также организовано их серийное производство и промышленное применение [2].

Несмотря на то, что уже на первом этапе работы в пользу этой гипотезы были получены разносторонние и весьма обнадеживающие экспериментальные данные [1] она безусловно нуждалась и нуждается в дальнейшей проработке и апробации.

Следует отметить, что такие исследования имеют и большую методическую привлекательность, которая состоит в следующем: последовательно проходя в процессе деструкции полимерной составляющей СОТС понижающиеся величины молекулярной массы, полимер тем самым проходит через различные физические состояния, уподобляясь на определенных этапах низкомолекулярным веществам, которые входят в состав СОТС в качестве основных присадок углеводородного происхождения. Следовательно результаты таких исследований могут быть использованы как при разработке СОТС на высокомолекулярной так и низкомолекулярной основах.

В этой связи в данной работе сделана попытка в гипотетических очертаниях хотя бы качественно увязать последовательно протекающие с большой скоростью превращения среды от исходной, физически активной, до химически активной, радикальной.

Превращение среды из физически активной в радикально активную обусловливается наложением и взаимодействием нескольких «внутренних» и «внешних» физических явлений, возникающих в зоне динамического контакта инструмента с обрабатываемым металлом. К ним следует отнести: высокую температуру, экзоэлектронную эмиссию, непрерывно образующуюся в результате разрыва межатомных сил связей при разрушении металла чистую, каталитически активную поверхность, возникновение термотока в системе замкнутой электрической цепи «станок -инструмент обрабатываемый металл» и конечно же, многообразный ассортимент СОТС, отличающийся разнообразием химического состава. Отметим, что ассортимент СОТС насчитывает около 200 наименований, представляющих собой многокомпонентные системы, содержащие комбинацию присадок различного химического состава, определяющие их различное функциональное и вспомогательное назначение.

Поэтому познание законов такого исключительно сложного по своей многоплановости и экстремальности условий протекания процесса, каковым является процесс резания металла при непрерывном поливе жидкости, содержащей несколько десятков компонентов, чрезвычайно затруднено. В связи с этим исследования, в рамках данной работы, были ограниченны и сконцентрированы главным образом на детальном изучении факторов, кардинально изменивших активность СОТС, в результате чего, как нам представляется, возможно получить предельно максимальный эффект от влияния СОТС на процесс резания или вообще на разрушение твердого тела. 


\section{Изложение основного материала исследования}

Эти же обстоятельства ограничили и выбор полимера в качестве присадки к СОТС. Исследовались алифатические предельные полимеры: полиэтилен (- $\left.\mathrm{CH}_{2}-\mathrm{CHCl}-\right)$. Их выбор обусловлен тем, что они дают возможность проследить изменение механизма влияния среды при неизменном ее химическом составе, но с возрастающей молекулярной массой от низкомолекулярных соединений (гептан) до высокомолекулярных (полиэтилен) с молекулярной массой, превышающей 100 . $10^{3}$. С другой стороны полиэтилен (ПЭ), являясь простейшим органическим полимером и обладая невысокой термостойкостью легко деструктирует с образованием активных форм углерода и водорода [7] высокая концентрация, которых в области разрушения, по-видимому, влияет на перестройку и разрыв сил связей между атомами, что должно вызывать, по сравнению с низкомолекулярным аналогом (гептан), скачкообразное снижение прочности.

Что касается поливинилхлорида (ПВХ), то он, кроме углерода, и хлорированного водорода, дает еще хлор, который является хорошим антисептиком и должен способствовать повышению устойчивости СОТС к различным грибковым заболеваниям.

Основываясь на перечисленных соображениях было выделено и изучались только основные процессы активации среды на примере ПЭ и ПВХ, как наиболее перспективные полимеры для применения в качестве добавок к СОТС, или для разработки новых СОТС на их основе.

Теплота является одним из основных физических показателей процесса резания. Её основными источниками являются пластическая деформация, происходящая прежде всего в зоне максимальных сдвигов, и трение в зонах контакта инструмента со стружкой и заготовкой.

Металлические поверхности, на которых происходит конденсация СОТС, имеют различную температуру, изменяющуюся в зависимости от вида и параметров механической обработки, а также свойств обрабатываемого металла в широком диапазоне от комнатной, а иногда, практически до температуры плавления обрабатываемого металла.

Известно, что с повышением температуры возрастает интенсивность всех видов молекулярного движения, что приводит к разложению химических соединений.

Вещество с большей молекулярной массой, но одного химического состава теряет свою устойчивость при более низкой температуре. Например, ПЭ высокого давления термоустойчив приблизительно до температуры $290^{\circ} \mathrm{C}$, с последующим выделением значительных количеств летучих продуктов, а низкомолекулярные линейные углеводороды теряют устойчивость только при температуре более 600С [7]. Если сопоставить энергию отрыва атома водорода в ряду насыщенных углеводородов, то в сравнительных единицах она составит для гептана 11, а для полимера, например, полиэтилена 3 , поливинилхлорида 2 единицы[8]. Основная причина такого различия состоит в том, что в полимерных молекулах часто встречаются термолабильные структурные аномалии, на которых могут легко инициироваться как типичные реакции, так и иные, совершенно неожиданные процессы. Кроме этого, многие реакции, типичные для низкомолекулярных соединений, превращаются в полимерах в ценные процессы, что обусловливается специфическим строением полимеров. Существенное отличие в механизме химических реакций низкомолекулярных и высокомолекулярных соединений обеспечивает, по-видимому, многократное превышение концентрации радикально активных, обладающих электрическим зарядом частиц водорода и приводит к повышению эффективности СОТС при введении в его состав полимера.

Следует отметить, что устойчивость полимеров к нагреванию, скорость их термического распада и характер образования продуктов зависит от химического строения цепи полимера. Однако первой стадией процесса всегда является образование макрорадикалов в результате разрыва наиболее напряженных и ослабленных связей в макромолекуле. В дальнейшем макрорадикалы вовлекаются в реакцию передачи цепи, т.е. передачи неспаренного электрона с образованием нового свободного радикала и макромолекулы с пониженной молекулярной массой Установлено, что каждый радикал способен инициировать радикальную реакцию, приводящую к разрыву около 10000 полимерных цепей [10].. Так например[9], для ПВХ реакция идет по следующему направлению:<smiles>[R][Y]C[C+]=C(Cl)C[R]</smiles>

Одновременно с этим происходит отщепление боковой группы $(\mathrm{Cl})$ от основной цепи с образованием двойных связей в цепи и выделением галоген - водорода: 


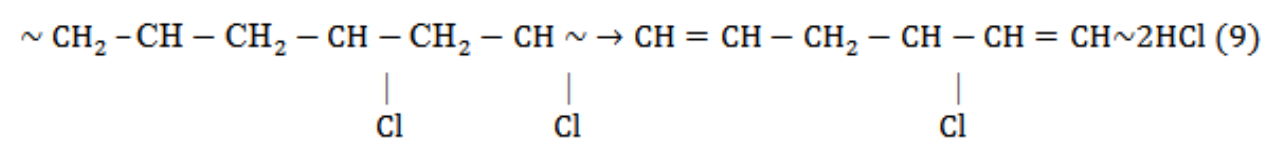

Вероятность протекания выборочных реакций зависит, конечно, от реакционной способности реактивов и взаимодействующих с ними молекул, а также от возможности столкновений реагирующих частиц.

Радикалы перемещаются от одной цепи (или фрагмента цепи) к другой путем отрыва атома водорода до тех пор, пока не сблизятся достаточно для того, чтобы произошла реакция.

Для (ПЭ) этот процесс схематически можно представить следующим образом[12]. Сначала происходит образование первичных радикалов при разрыве молекул $-\mathrm{CH}_{2}-\mathrm{CH}_{2} \bullet$ (I)

Первичные радикалы легко реагируют с молекулами П.Э., отрывая водород с образованием серединных радикалов. Отрыв водорода может привести к образованию радикалов трех типов:

$$
-\mathrm{CH}_{2}-\dot{\mathrm{C}} \mathrm{H}-\mathrm{CH}_{2}-\text { (II) } ;-\mathrm{CH}_{2}-\mathrm{C}-\mathrm{CH}_{2}-\text { (III) } ;-\underset{\mathrm{R}}{\stackrel{\mathrm{C}}{\mathrm{CH}_{2}}-\mathrm{CH}}-\mathrm{CH}_{2}-\text { (IV) }
$$

Эти радикалы далее реагируют следующим образом:

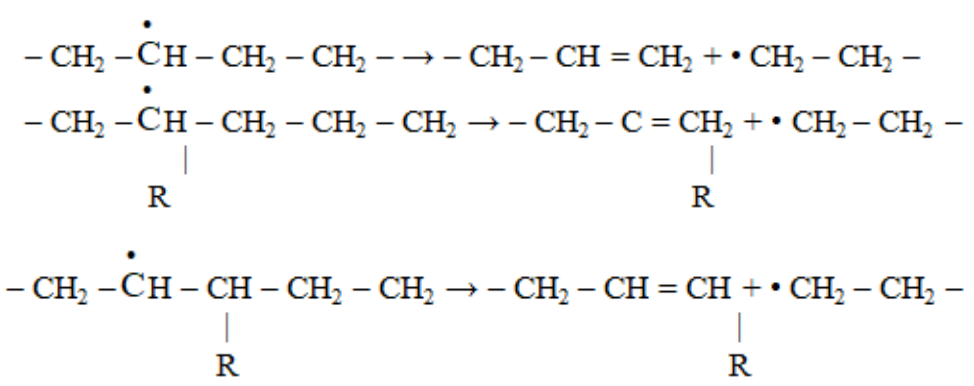

Образующиеся концевые макрорадикалы в свою очередь реагируют с макромолекулами, что сопровождается распределением энергии вдоль цепей. В приведенной схеме число разрывов значительно превышает количество макрорадикалов, образовавшихся непосредственно при воздействии температуры.

На основании изложенного, деструкцию ПЭ следует представлять как процесс, который развивается путем непрерывного разрыва и рекомбинации макромолекул с образованием разветвлений и поперечных связей. При этом в зоне химической реакции повышается температура и образуется водород.

Следовательно, согласно развиваемой модели деструкция полимеров, которые можно рекомендовать в качестве присадки к СОТС должна быть ценным радикальным процессом. Это только одно из основных требований, которое обеспечит высокую концентрацию водорода в зоне разрушения.

Проведенные исследования в этом направлении [1] показали, что при введении ПЭ или ПВХ в СОТС, или в воду и применяя ее, как модель СОТС, в зоне резания образуется газовая смесь, состоящая из химических элементов, входящих в состав в цепи полимера в различных сочетаниях от $\mathrm{C}$ до $\mathrm{C}_{12}$ и водорода (ПЭ), а также водорода и хлористого водорода (ПВХ).

В процессе механической обработки стали на режущих кромках инструментов накапливалась также углеродистая масса, как продукт распада ПЭ и ПВХ. Углеродистая масса, разделяя трущиеся поверхности значительно снижает трение, и кроме этого, углерод диффундируя в режущие кромки инструмента образует твердые карбиды, способствуя тем самым повышению износостойкости инструмента [1].

При чем, количество водорода в газовой смеси было очень значительным и иногда достигало $90 \%[11,12]$.

Таким образом, при термодеструкции полимерной присадки СОТС на нагретых поверхностях металла, на промежуточных стадиях химических реакций образуются продукты, обладающие чрезвычайно высокой химической активностью, а именно: водород в активных формах, радикалы и относительно стабильные соединения.

Н.Н. Семеновым было установлено [13], что образование таких промежуточных продуктов высокой активности является дополнительным источником активных частиц и реакция приобретает 
самоускоряющийся характер. В результате самоускоряющейся цепной реакции, протекающей даже в изотермических условиях, происходит образование больших концентраций активных частиц, составляющих водородную плазму.

Расчеты, приведенные в работе[14] показывают, что количество протонов, атомов и радикалов, а следовательно и скорость реакции в этом случае возрастает по экспоненциальному закону е фактор разветвления, определяемый разностью между вероятностью разветвления цепи и её обрыва на поверхности нагретого металла, с которым контактирует макроцепь полимера.

Здесь важно подчеркнуть, что промежуточные вещества не приводят к неограниченному развитию цепной реакции в связи с тем, что на определенной стадии она обрывается вследствие гибели активных центров в результате их адсорбции на металле или соударений между собой в объеме смеси.

Наиболее важно однако, что первые акты разрыва полимерных молекул длинною порядка сотни ангстрем кроме того, что они инициируют химические свободно радикальные процессы [15,16], большая часть (примерно 80\%) запасенной в «половинах» разорвавшегося фрагмента энергии выделяется в виде тепла[17]. Учитывая упоминавшийся выше цепной характер деструктивных процессов, когда вслед за распадом первой молекулы передача свободных радикалов ведет к быстрому распаду сотен соседних молекул[18], и следовательно, к «групповому» тепловыделению, можно заключить, что, в объеме такого полимера протекают своеобразные тепловые микровзрывы.

В качестве примера приведем реакцию молекул углеводородов с атомами или радикалами, когда выделяющаяся энергия превышает более чем на порядок затраченную на реакцию $[14,15]$ :

$$
\begin{gathered}
\mathrm{H}+\mathrm{Cl}_{2} \rightarrow \mathrm{HCl}+\mathrm{Cl}(1) \\
\mathrm{H}+\mathrm{C}_{2} \mathrm{H}_{6} \rightarrow \mathrm{H}_{2}+\mathrm{C}_{2} \mathrm{H}_{5}(2)
\end{gathered}
$$

Для реакции (1) необходимо затратить 2 ккал/моль, а освобождается 57 ккал/моль. Для второго случая, для реакции необходимо затратить 9,5 ккал/моль, а освобождается 98 ккал/моль [21].

Вместе с тем взаимодействие между частицами нерадикальной природы при разрыве связи не происходит значительно выделение энергии.

В этом случае требуется преодоление высокого энергетического барьера и химическая реакция не может осуществляться с заметной скоростью. Например, для реакции $\mathrm{H}_{2}+\mathrm{I}_{2} \rightarrow$ НІ затрачивается 40 ккал/моль, а образуется 138 ккал/моль .

При контакте низкомолекулярной органической молекулы с нагретой металлической поверхностью в зависимости от ее природы, состояния металлической поверхности и температуры, контакт молекулы может быть не эффективным даже при очень высокой температуре (около 1900 드), а в других условиях такой распад молекулы происходит с малой скоростью .

При столкновении молекул $\mathrm{C}_{2} \mathrm{H}_{2}$ с металлом (вольфрамом) она прилипает к металлу только, когда металл нагрет до высокой температуры $(1000 \mathrm{C})$. В этом случае один из углеродных атомов молекулы образует связь с металлом, что приводит к локальному образованию карбида, другие атомы молекулы (водород) немедленно перераспределяются между собой и вылетают в объем в виде более простых молекул (например $\mathrm{H}_{2}$ в случае $\mathrm{C}_{2} \mathrm{H}_{2}$ или $\mathrm{H}_{2}$ и $\mathrm{CO}$ в случае $\mathrm{CH}_{3} \mathrm{CHO}$ или $\mathrm{CH}_{3} \mathrm{COCH}_{3}$ ); свободные радикалы при этом не образуются .

Таким образом микровзрывы, которые являются постоянным спутником химической активации полимерной составляющей СОТС, сопровождаются выделением тепла и увеличением внутренней энергии системы, что приведет к снижению суммарной энергии, необходимой для разрушения твердого тела, т.е. будет способствовать снижению энергозатрат на процесс резания.

Инициировать и поддерживать ход химических превращений полимерной присадки в процессе резания металла может также экзоэлектронная эмиссия.

Известно $[10,14]$ что при разрушении металла, особенно в процессе резания, излучается поток электронов достигаемый $6 \cdot 10^{3}$ импульсов в минуту, а их энергия составляет, примерно, $10^{2}$ эв.

Поскольку энергия химических связей в полимерах соответствует 2,5-4,0 эв , то такое излучение способно вызвать разрыв связей основной макроцепи полимера, отрыв замещающих групп, сшивание и др. В отличии от термодеструкции реакции вызванные лучистой энергией не является цепным процессом. Под действием излучений молекулы полимера ионизуются и возбуждаются. Возбужденная молекула распадается на радикалы, а выделяющейся при этом вторичный электрон - рекомбинирует с образованием иона полимера, а также взаимодействует с другими молекулами, образуя новые ионы.

Примечательно, что также, как при воздействии температуры основным летучим продуктом при воздействии потока электронов на полиэтилен является водород, а на поливинилхлорид-хлорид водорода, хлор и водород [1].

Таким образом, становится ясно, что влияние температуры в зоне резания, как и экзоэлектронной эмиссии направлено на деструкцию макроцепей полимерной присадки СОТС, образование промежуточных активных продуктов. 
Даже беглый и, естественно, неполный анализ проблем, которые исследуются на основе изучения систем жидкая или газовая среда - деформируемый металл дает основание полагать, что в дальнейшем, химические преобразования участников реакций продолжаются и заканчиваются на свежеобразованных поверхностях металла в результате его разрушения. Этот вывод также подтверждается следующими экспериментальными данными.

Известно [14], что механическое разрушение твердых тел является одним из способов получения ювелирной, чистой, каталитически активной поверхности. Такая поверхность обычно обладает повышенной химической активностью обусловленной присутствием различных типов активных центров - атомов, радикалов, некомпенсированных или напряженных сил связей между атомами. При этом, для протекания на таких поверхностях каталитической реакции важное значение имеет также и структура поверхности, т.е. тип симметрии и пространственное расположение атомов на поверхности, а также её «вторичная» структура- ступеньки, ребра и другие микротопологические особенности [12].

Изучение хемесорбции и простых химических реакций на поверхности, образованной в результате разрушения металла, показало [11], что при этом достигается весьма значительное повышение химической активности адсорбированных молекул. Так, например хемасорбция молекул водорода, азота и кислорода приводит к их диссоциации с образованием атомов и ионов даже при температуре $-188^{\circ} \mathrm{C}$ (в некоторых случаях и при температуре сжижения водорода $\left.-253^{\circ} \mathrm{C}\right)$. Диссоциация протекает очень быстро, практически без энергии активации.

Способность такой поверхности генерировать в объем свободные атомы, а также заряженные частицы водорода $\left(\mathrm{H}^{+}\right.$и $\left.\mathrm{H}^{-}\right)$, особенно с повышением температуры, известна со времен ранних робот Ленгмюра, на основе которых был создан один из простых методов получения водорода в активных формах, а процесс этот идет по следующей двухстадийной схеме [12]:

$$
\begin{gathered}
\mathrm{Me}+\mathrm{H}_{2} \rightarrow \mathrm{Me}-\mathrm{H}+\mathrm{H} \\
\mathrm{Me}-\mathrm{H} \rightarrow \mathrm{Me}+\mathrm{H}
\end{gathered}
$$

Низкомолекулярные углеводородные продукты, образующиеся в процессе химического преобразования среды, также дают атомарный водород. Например [11] молекула метана $\mathrm{CH}_{4}$, которая образуется в процессе преобразования цепи полимера, сначала превращается в молекулярный ион $\mathrm{CH}_{4}^{+}, \mathrm{a}$ затем в ион металла $\mathrm{CH}_{3}^{+}$и атомный водород:

$$
\mathrm{CH}_{4} \rightarrow \mathrm{CH}_{4}^{+}+\mathrm{e} ; \mathrm{CH}_{4} \rightarrow \mathrm{CH}_{3}^{+}+\mathrm{H}
$$

В работе [12] показано, что молекула $\mathrm{C}_{2} \mathrm{H}_{2}$ хемосорбируется при нормальных условиях на чистом железе одним из своих углеродистых атомов, что приводит к локальному образованию карбида и ионизированного водорода.

В дальнейшем водородный атом может действовать как донор электрона $\left(\mathrm{H} \rightarrow \mathrm{H}^{+}+\mathrm{e}^{-}\right)$, а может участвовать в реакциях, отрывая второй водородный атом из гидратной оболочки иона металла [16]:

$$
\mathrm{Fe}\left(\mathrm{H}_{2} \mathrm{O}\right)_{6}^{2+}+\mathrm{H} \rightarrow \mathrm{Fe}^{3}+\mathrm{OH}^{-}+\mathrm{H}_{2}
$$

Ионизация атомов водорода на каталитической поверхности может идти несколькими путями и заканчивается образованием $\mathrm{H}^{-}$и $\mathrm{H}^{+}$. Если интенсивность силового поля недостаточна для полной ионизации водорода до протона, то атом водорода будет находиться в состоянии возбуждения и частичной ионизации.

Следует отметить, что положительные ионы обладают более высокой реакционной способностью, чем отрицательные. Простейшая реакция, которая идет с высокой скоростью передачи заряда (т.е. электрона) по следующей схеме [12,13]:

$$
\begin{gathered}
H^{+}+\mathrm{CH}_{4} \rightarrow H^{\bullet}+\mathrm{CH}_{4}^{+}, \text {a также } \mathrm{CH}_{4}^{+}+\mathrm{CH}_{4} \rightarrow \mathrm{CH}_{5}^{+}+\bullet \mathrm{CH}_{3} \bullet \\
\mathrm{CH}_{3}^{+}+\mathrm{CH}_{4} \rightarrow \mathrm{C}_{2} \mathrm{H}_{5}^{+}+\mathrm{H}_{2}^{\bullet}
\end{gathered}
$$

К наиболее важным реакциям ионов на поверхности металла следует отнести также возможную их внутреннюю перестройку и разложение, что приводит к образованию новых ионов, молекулярных продуктов и свободных радикалов. При этом, такие процессы, протекающие от момента образования первичных ионов завершаются всего за $10^{-5}$ сек .

Параллельно с такими процессами некоторые ионы претерпевают нейтрализацию, обычно сопровождающуюся выделением значительного количества энергии. Вследствие этого продукты нейтрализации находятся в состоянии высокого возбуждения и способны участвовать в дальнейших реакциях, активируя даже молекулы исходного вещества или его промежуточные продукты, образующие 
в процессе химических реакций. Необходимо отметить [13], что в этом случае выделяется значительное количество энергии, что должно влиять на тепловые функции атомов твердого тела, снижая, как уже отмечалось, усилия, необходимые для разрыва сил межатомных связей.

Известно $[17,18]$, что каталитически активная поверхность металла не остается в процессе реакций химически неизменной. В действительности меняется не только состояние ее поверхности, но и в результате протекания самой реакции и адсорбции на ней атомов реагирующего вещества, ухудшается ее каталитическая активность. Это означает, что в нашем случае, при катализе на поверхности металла низкомолекулярных осколков макроцепи полимера, низкомолекулярных углеводородных молекул и др. На поверхности должен находится адсорбированный слой углеродных атомов. В связи с этим, методом ОЖЕ спектроскопии исследовалась поверхность металлов (ст. 45, $\mathrm{Fe}, \mathrm{Ti}, \mathrm{Co}, \mathrm{Ni}, \mathrm{Mo}$ ) после точения в СОТС с добавкой 1,5\% ПЭ [1]. Установлено, что поверхности всех исследуемых металлов примерно, на 80-90\% покрыта углеродом, концентрация которого непрерывно снижается по мере удаления от поверхности. И на глубине N $1000 \AA ̊$ химсостав материала становится равным его исходному состоянию. На поверхности образцов после точения в СОТС без полимера также обнаруживался углерод, однако концентрация его незначительна и составляла всего 8-12 атомных процентов.

Согласно гипотезы решающую роль в облегчении процессов деформации и разрушения в СОТС играет водород. Косвенным доказательством этого являются вышеизложенные данные об образовании водорода в зоне разрушения металла при его резании в СОТС. Поэтому, если гипотеза верна, то водород должен присутствовать в стружке и обработанной поверхности.

Для обнаружения водорода был применен метод температурно-программируемого нагрева образца (стружки или срез обрабатываемой поверхности), помещенного в вакуумный объем, с одновременной масс-спектрометрической регистрацией выделяющего водорода [16], если он действительно содержится в образце. Полученные при этом экспериментальные данные представляют собой зависимости сигнала масс-спектрометра на заданной массе (пропорционально скорости выхода водорода из образца) от температуры образца, которая поднималась со скоростью 0,5 к/с.

\section{Выводы}

Установлено [1], во всех образцах полученных в результате резания стали в СОТС с полимером регистрировался четкий сигнал масс- спектрометра, что соответствовало десорбции водорода из образца с повышением его температуры. В образцах после резания в СОТС без полимера также обнаруживался водород, однако его концентрация была значительно меньше, чем в образцах после обработки в СОТС с полимером.

Важно также отметить, что водород был зарегистрирован в образцах стали после их испытании в СОТС с полимером и без полимера на статическое растяжение и сжатие. А при испытании в тяжелой воде $D_{2} \mathrm{O}$ в образцах обнаруживался дейтерий.

Таким образом становится ясно, что постоянные спутники процесса резания температуры, эмиссия электронов, каталитически активная поверхность разрушаемого металла оказывая комплексное влияние на поверхностно-активную жидкость, переводится в новое, радикальное состояние водородную плазму.

При этом химическая активация среды особенно ярко выражена, когда в жидкость вводится определенного состава строения и концентрации высокомолекулярное соединение. В этом случае макромолекулы полимера в составе СОТС не только значительно увеличивают выход конечного продукта химических реакций - водорода в активных формах, но и образуют на промежуточных химических реакциях атомы водорода, радикалы и относительно стабильные соединения, являющиеся дополнительным источником образования большой концентрации активных частиц.

Обширный экспериментальный материал, накопленный в такого рода исследованиях, аргументы приведенные в данной статье, дают основание считать, что адсорбция поверхностно-активной среды на деформируемом металле вовсе не является определяющим фактором в облегчении процесса деформации и разрушения, а только первым, хотя и чрезвычайно важным этапом, на пути многостадийных химических преобразований среды. Решающую роль в понижении прочности металлов в адсорбционных средах играют активные формы водорода.

\section{Список использованной литературы}

1. Сошко А. И. Смазочно-охлаждающие средства в механической обработке металлов. / Сошко В.А. - Херсон: Изд. Олди-плюс, 2008. - 388 с.

2. Лихтман В.Н. Физико-химическая механика материалов. / Щукин Е.Д., Ребиндер П.А. - М.: Изд. АНСССР, 1962. - 277 c.

3. Тугов И.И. Химия и физика полимеров. / Кострыкина Г.И. - М.: Изд. Химия, 1989, -430 с.

4. Кулезнев В.Н. Химия и физика полимеров. / Шершнев В. М., Изд. Высшая школа, 1988. - 311 с.

5. Справочник. Смазочно-охлаждающие средства для механической обработки металлов резанием, под ред. Энтелиса С.Г., М: Машиностроение, 1986. - 352 с. 
6. Налбандян А.Б. Химическая кинетика и ценные реакции. К 70-летию академика Н.И. Семенова. - М.: Изд. Наука, 1966. - 603 с.

7. Семенов Н.И. О некоторых проблемах химической кинетики и реакционной особенности. -М.: Изд. Анесср, 1958. -317 c.

8. Корчин В.А., Слонимский Г.Л. ДАНСССР, 105, 1955. - 751 с.

9. Слонимский Г.Л. ЖВХО, 4, 1959, -73 с.

10. Годовский Ю.К. Ср ТТ / Панков В.С., Слуцкер А.И. 13, №8, 1971, - 2289 с.

11. Soshko, V.A., Siminchenko, I.P. Transformation of the surface-active lubricating and cooling working media into the active radical forms in the cutting zone. Fizyko-Khimichna Mekhanika Materialiv, Vol. 51, No. 2, pp. 72-76, March-April, 2015. DOI: 10.1007/s11003-015-9833-9

12. Химические реакции полимеров. /Под ред. Е. Феттеса. - М.: Изд. «Мир», 1967. - 2537 с.

13. Сабо 3.Г. Сб. Химическая кинетика и ценные реакции. М.: Наука, 1966, - 46 с.

14. Адамсон А.А. Физическая химия поверхностей. - М.: Мир, 179, - 567 с.

15. Ахматов А.С. Молекулярная физика граничного трения. - Физматгиз, 1962. - 472c.

16. Рогинский С.3. Строение вещества и спектропия, - М: Изд. АН СССР, 1960. - 217 с.

17. Рабинович Б.С. Химическая активация / Рабинович Б.С., Флауэрс Н.С. - М: Наука, 1966. - 61 с.

18. Закревский В.А. Высокомолекулярные соединения / Закревский В.А. - М: Изд. Химия, 1971. - 105с.

\section{References}

1. Soshko A. I. Lubricant-cooling means in the machining of metals. / Soshko V.A. Ed. Oldie-plus, Kherson, 2008. $-388 \mathrm{~s}$.

2. Likhtman V.N. Physico-chemical mechanics of materials. / Schukin E.D., Rebinder P.A. M., ed. ANSSSR, 1962. - 277 p.

3. Tugov I.I. Chemistry and physics of polymers. / Kostrykina G.I. M., ed. Chemistry, 1989. - $430 \mathrm{~s}$.

4. Kuleznev V.N. Chemistry and physics of polymers. / Shershnev V.M., Ed. High School, 1988. - 311 pp.

5. Reference. Cutting lubricants for the mechanical processing of metals by cutting, ed. Entelisa S.G., M: Mechanical Engineering, 1986. - 352 p.

6. Nalbandyan A.B. Chemical kinetics and valuable reactions. To the 70th anniversary of academician N.I. Semenova. Ed. Science, M., 1966. - 603 p.

7. Semenov N.I. About some problems of chemical kinetics and reaction features. M., ed. Anessre, 1958, $317 \mathrm{p}$.

8. Korchin V.A., Slonimsky G.L. DANSSSR, 105, 1955. - 751 s.

9. Slonimsky G.L. ZhVHO, 4, 1959. -73 p.

10. Godovsky Yu.K. Sr TT / Pankov V.S., Slutsker A.I. 13, No. 8, 1971. -2289 p.

11. Soshko, V.A., Siminchenko, I.P. Transformation of the surface-active lubricating and cooling working media into the active radical forms in the cutting zone. Fizyko-Khimichna Mekhanika Materialiv, Vol. 51, No. 2, pp. 72-76, March - April, 2015. DOI: 10.1007 / s11003-015-9833-9

12. E. Fettes. Chemical reactions of polymers. Ed., Ed. "World", Moscow, 1967, t.2. -537 pp.

13. Clogs Z.G. Sat Chemical kinetics and valuable reactions. M., Science, 1966.- 46 p.

14. Adamson A.A. Physical chemistry of surfaces. Moscow: Mir, 179. -567 p.

15. Akhmatov A.S. Molecular physics of boundary friction. Fizmatgiz, 1962, 472s.

16. Roginsky S.Z. The structure of matter and spectropy, M: Izd. USSR Academy of Sciences, 1960. -217 p.

17. Rabinovich B.S. Chemical activation / Rabinovich B.S., Flowers N.S. - M: Nauka, 1966. -61 p.

18. Zakrevsky V.A. High-molecular compounds / Zakrevsky V.A. - M: Publ. Chemistry, 1971. -105c. 